$\begin{array}{cl}\begin{array}{c}\text { Revue } \\ \text { de } / \text { histoire }\end{array} & \text { Revue de l'histoire des religions } \\ \text { des religions } & \begin{array}{l}3 \mid 2005 \\ \text { Varia }\end{array}\end{array}$

\title{
Lire Reuchlin lire la Bible
}

Sur la préface des Rudimenta hebraica (1506)

Read Reuchlin Reading the Bible: on the Foreword of Rudimenta Hebraica (1506)

Jean-Christophe Saladin

\section{OpenEdition Journals}

Édition électronique

URL : http://journals.openedition.org/rhr/4188

DOI : 10.4000/rhr.4188

ISSN : 2105-2573

Éditeur

Armand Colin

Édition imprimée

Date de publication : 1 juillet 2005

Pagination : 287-320

ISBN : 2200-92086-5

ISSN : 0035-1423

Référence électronique

Jean-Christophe Saladin, « Lire Reuchlin lire la Bible », Revue de I'histoire des religions [En ligne]

3 | 2005, mis en ligne le 19 janvier 2010, consulté le 01 mai 2019. URL : http://

journals.openedition.org/rhr/4188; DOI : 10.4000/rhr.4188 


\section{Lire Reuchlin lire la Bible Sur la préface des Rudimenta hebraica (1506)}

En 1506, Jean Reuchlin, pionnier de l'humanisme allemand, publiait ses Rudimenta hebraica, un manuel d'hébreu destiné aux chrétiens, pour leur permettre d'étudier la Bible dans sa langue originelle. Il justifiait ce projet par une lettre-préface qui entraîne le lecteur dans un labyrinthe de métaphores et de références croisées particulièrement complexes. Nous nous efforçons de décrypter ce jeu littéraire et de mettre au jour le sens implicite de ces formules, souvent elliptiques ou masquées par leur usage conventionnel. Cette approche souligne à quel point ses relations à la Bible et aux juifs purent paraître provocatrices à nombre de ses contemporains.

\section{Read Reuchlin Reading the Bible : on the Foreword of Rudimenta Hebraica (1506)}

In 1506 Jean Reuchlin, pioneer of German humanism, published his Rudimenta hebraica, a Hebrew handbook intended for Christians, to allow them to study the Bible in its original language. He justified this project by a letter-foreword which involves the reader in a labyrinth of particularly complex metaphors and cross-references. We endeavour to decipher this literary game and to bring to light the implicit meaning of these expressions, often elliptic or masked by their conventional use. This approach underlines to what extent his relationship with the Bible and the Jews could appear provocative to many of his contemporaries. 


\section{Exegi monumentum ære \\ Perennius Nonis \\ Martiis Anno \\ MD.VI.}

«J'ai élevé un monument plus durable que le bronze, le 7 mars 1506. » C'est en ces termes pompeusement empruntés à Horace ${ }^{1}$, que l'humaniste Jean Reuchlin signa la dernière page de ses Rudimenta hebraica ${ }^{2}$.

Cette citation peut être lue comme une simple afféterie d'auteur, qui n'a rien pour surprendre sous la plume d'un humaniste, se plaisant à se représenter comme un nouvel Horace. Cependant, le lecteur « cultivé », se souvenant que le «monument plus durable que le bronze » d'Horace n'est autre que son œuvre poétique, observera que le gros livre de Reuchlin (un in-folio de 624 pages) n'est pas un recueil d'odes ni de satires, mais une méthode d'hébreu - la première composée par un non-juif en Occident ${ }^{3}$. Vu le sujet traité, l'image semble donc assez incongrue, car on se serait plutôt attendu à voir son auteur se comparer à saint Jérôme ou à quelque autre grand philologue de l'Antiquité.

\section{LA MISE EN PAGE « HÉBRAÏQUE »}

Ce vers latin mérite-t-il donc qu'on lui accorde plus d'attention qu'à tout autre ornement littéraire imposé par le goût du temps ? La plupart des modernes ne le penseront pas. Reuchlin lui a pourtant

1. Horace, Odes, III, 30, 1.

2. L'ouvrage fut publié chez Thomas Anshelm à Pforzheim sous le titre : Ioannis Revchlin Phorcensis $l l$. Doc ad dionysium fratrem suum germanum de rudimentis hebraicis. Il en existe trois exemplaires à la Bibliothèque de l'Arsenal à Paris et il peut être consulté sur le site Internet Gallica de la Bibliothèque nationale de France. C'est à cette édition que je me référerai au cours de l'article. Une réédition en fac-similé en a été publiée en $1974 \mathrm{chez}$ Georg Olms, Hildesheim. Sa lettre-préface avait été éditée séparément (d'après l'édition de Henri Petri à Bâle, 1537), avec une partie de la correspondance de Reuchlin, par Ludwig Geiger dans Johann Reuchlins Briefwechsel, Stuttgart, 1875, p. 88-101, reproduit chez Georg Olms, Hildesheim, 1962.

3. Si l'on excepte le De modo legendi et intelligendi hebrceum, fascicule de 42 pages publié par son élève Konrad Pellikan en 1504 à Strasbourg. 
réservé une place de choix dans la « mise en scène » typographique de son ouvrage. En effet, bien que ce cul-de-lampe constitue les derniers mots de son texte, il trône au milieu de la première page car le livre doit s'ouvrir «à l'envers », selon l'usage de l'hébreu. Cependant, le lecteur «latin» ignore ce détail tant qu'il n'a pas tourné les premières pages du livre. Ainsi, quand il ouvre le livre selon l'usage «latin », il trouve d'abord une page (de droite) sur laquelle il peut lire les mots Finis libri (« fin du livre»), suivis des quelques vers suivants :

\section{RÈGLE}

Ce livre ne doit pas se lire comme les autres,

tiens la face de la main gauche et le dos de la main droite, et tourne chaque page de la main gauche vers la droite.

Celles que tu trouveras en latin,

lis-les à la façon latine, mais quand il y en a en hébreu,

Il faut les lire de droite à gauche ${ }^{4}$.

Après cette annonce, le lecteur qui tourne la page découvre (en page de droite à nouveau) les fières armoiries de Reuchlin, surmontées de ses titres ${ }^{5}$. S'il tourne encore, il trouve cette fois deux pages imprimées face-à-face. Sur celle de gauche, il lira un éloge de Reuchlin rédigé par Georges Simler et adressé à l'imprimeur Thomas Anshelm, daté du 27 mars 1506, et sur la page de droite enfin, la dernière page du texte de Reuchlin, donc de l'ouvrage proprement dit (p. 621, non foliotée), dont la citation d'Horace occupe la place centrale. C'est seulement à ce moment que le lecteur comprend vraiment que le livre est imprimé « à l'envers » et qu'il faut le lire de droite à gauche. S'il reprend alors le livre par l'autre extrémité, il trouve la vraie première page, sur laquelle est écrit : Principium libri (« début du livre »). S'il la tourne dans le sens «hébraïque », il trouvera enfin le vrai début du texte (folioté p. 1).

La présence du blason sur la première (dernière) page lui donne une allure de frontispice destiné aux lecteurs «latins», aucun

4. Finis libri/ Canon / Non est liber legendus hic seu cateri / Faciem sinistra dextera dorsum tene / Et de sinistra paginas ad dexteram / Quascumque uerte.qua latina uideris / Legito latine hebraea si sint insita / A dextera legenda sunt sinistrorsum (Rudimenta, p. 625, non foliotée).

5. Reuchlin (1455-1522) avait été anobli par Frédéric III en 1493. 
imprimeur n'ornant d'aussi belles armoiries la dernière page d'un livre. Grâce à cette astuce de présentation, la citation d'Horace occupe bien la place centrale dans l'ouverture du livre. On sera donc d'autant plus surpris de lire une condamnation sévère des poètes dès les premières lignes du texte de Reuchlin. J'y reviendrai.

Il faut maintenant dire un mot sur la construction globale de ce manuel d'hébreu. Il est composé de trois parties (trois « livres»), dont les livres I et II sont un dictionnaire (De elementis) et le livre III une grammaire (De oratione et eius partibus), le tout réuni dans un seul volume. Voici en quels termes l'auteur les présente :

« Je composerai donc l'ouvrage complet en trois volumes, avec l'aide de Dieu. Le premier traitera des lettres, des syllabes et des mots, jusqu'aux mots qui commencent par $\mathrm{K}$, le second des mots qui commencent par L jusqu'à la fin du dictionnaire, le troisième de l'art de la grammaire et de son apprentissage. Le titre de ce livre est : Les Rudiments hébraïques pour la raison que j'ai composé ces volumes à l'intention non pas de ceux qui sont déjà instruits, mais des ignorants et des étudiants. Ensuite, si Dieu y consent, j'en donnerai d'autres, qui seront consacrés à l'étude du secret de Pythagore et à la science kabbalistique, absolument incompréhensible à qui ne connaît pas l'hébreu. ${ }^{6}$ »

\section{LA LETTRE À SON FRÈRE DE SANG}

Reuchlin a enchâssé ce dictionnaire et cette grammaire entre les pages d'un long texte rédigé à la manière d'une lettre familière adressée à son frère Denis (Jo. Reuchlin Phorcensis LL Doc. ad Dionysium fratrem suum germanum de Rudimentis Hebraicis liber primus). Le début de la lettre sert ainsi de préface au premier livre. Elle s'interrompt à la fin de la quatrième page, puis reprend au

6. Tribus itaque voluminibus totum negotium, adjuvante Deo, conficiam: primum erit de literis, syllabis, dictionibus, usque ad ea vocabula, qua a $\mathrm{K}$ incipiunt, secundum de dictionibus inchoantibus ab L usque ad finem vocabularii, tertium de arte grammatica et ejus exercitatione. Inscriptio hujus libri est : De rudimentis hebraicis, eo quod non jam doctis, sed rudibus ac erudiendis ea volumina composuerim, deinceps altiora deo annuente daturus, qua ad arcance Pythagora disciplinam et artem Cabalisticam deserviunt, a nemine prorsus intellecta, nisi Hebraice pradocto (Rudimenta, p. 3 et 4). 
début du livre III (p. 546 à 549) pour se terminer à la fin du livre III (p. 620-621) où elle joue cette fois le rôle d'une postface à l'ensemble de l'ouvrage, en se terminant par la fameuse citation d'Horace.

Comme le veut l'usage, cette lettre-préface contient les déclarations d'intention de l'auteur et les justifications de son projet - et ce seul fait justifie déjà son intérêt. De plus, Reuchlin y relate avec nombre de détails les péripéties de sa formation intellectuelle à travers l'Europe de la fin du XV siècle, ce qui n'est pas d'un intérêt moindre étant donné l'importance du personnage. Je ne m'attarderai cependant pas à cet aspect car sa vie et ses œuvres ont déjà été largement étudiées par les historiens allemands, depuis Ludwig Geiger et Eduard Böcking au milieu du XIX ${ }^{\mathrm{e}}$ siècle, jusqu'à Max Brod, qui en publia une très riche biographie un an avant sa mort ${ }^{7}$.

Je laisserai également à d'autres le soin de parler du contenu proprement philologique de l'ouvrage et je me limiterai à sonder sa préface. Son écriture fort complexe est en effet truffée de métaphores et de formules obéissant au goût humaniste le plus convenu, que le lecteur considère généralement comme de simples ornements, mais dont la lecture attentive révèle des jeux d'allusions et de non-dits souvent lourds de significations. Je pense que c'est souvent par ce qu'il ne disait pas que Reuchlin se posa le plus scandaleusement à contre-courant de son époque - y compris parfois de ses amis -, ce qui contribua sans doute à irriter prodigieusement ses adversaires et à leur faire perdre toute mesure au cours des années de polémiques qui suivirent (que les Allemands nomment Bücherstreit).

7. Ludwig Geiger, Johann Reuchlin, sein Leben und seine Werke, Leipzig, Duncker \& Humboldt, 1871, repr. Nieuwkoop, 1964 ; Eduard Böcking a reproduit de nombreuses pièces importantes concernant l'Affaire Reuchlin, ainsi qu'une bibliographie commentée des sources, dans Ulrichi Hutteni Equitis Operum Supplementum, 2 volumes, Leipzig, Teubner, 1864-1870, rééd. Aalen, 1963 ; Max Brod, Johannes Reuchlin und sein Kampf, Stuttgart-Berlin-Cologne, W. Kohlhammer, 1965. On consultera aussi bien sûr Heinrich Graetz, qui consacre à Reuchlin une partie importante de sa Geschichte der Juden von den ältesten Zeiten bis auf die Gegenwart (Band IX : Geschichte der Juden von der Verbannung der Juden aus Spanien und Portugal..., Leipzig, 1866, rééd. Hirsch Arani, Berlin 1998) et Salo Wittmayer Baron, A Social and Political History of the Jews, $2^{\mathrm{e}}$ éd. augmentée, vol. 13, Columbia University Press, New York, 1969. 
On sait en effet la violence des attaques dont il fut l'objet quelques années plus tard, pour s'être opposé à la confiscation et à la destruction des livres des juifs de Cologne. Cette prise de position, qu'il rendit publique dans son Augenspiegel en 1511, déclencha une longue affaire juridico-religieuse (nommée negotium Reuchlini par Érasme) qui le mit aux prises avec le converso Pfefferkorn, soutenu par le Grand Inquisiteur Hochstraten et les dominicains de Cologne ${ }^{8}$, et se poursuivit jusqu'à sa mort en 1522. Malgré le soutien de Léon $\mathrm{X}$ et de nombreux humanistes, il fut finalement condamné en 1520 - une semaine après la fulmination de la bulle Exsurge Domine contre Luther, cette coïncidence n'étant certes pas fortuite. L'affaire donne un relief particulier à ses considérations de 1506 sur l'étude de l'hébreu.

En un mot, quelle justification Reuchlin donne-t-il à la publication de ce manuel d'hébreu ? Tout simplement la nécessité pour les chrétiens de lire la Bible dans sa langue originelle - en y ajoutant, il est vrai, que la lecture habituelle de la Bible à la façon de son temps est devenue proprement « repoussante (explosa).

«Puisque la trop grande familiarité de leur lecture quotidienne les a rendues repoussantes, nous trouverons nous-mêmes la manière nouvelle et originelle de dire les divines écritures, telles qu'elles ont été prononcées par la bouche de Dieu, et cela nous remplira assurément d'une admiration particulière pour cette étude inouïe tout autant que récente. ${ }^{9}$ »

Cette thèse, qui ne choquera guère un lecteur moderne, était cependant fort provocatrice à l'époque où elle fut publiée (onze ans avant l'affichage des 95 thèses de Luther). D'autant que les persécutions contre les juifs battaient leur plein et que l'accusation de « judaïsation clandestine » pouvait aisément conduire au bûcher.

8. On trouvera une analyse de cette affaire dans mon édition des Lettres des hommes obscurs, d'Ulrich von Hutten, Paris, Les Belles Lettres, 2004.

9. [...] ut nimia familiaritate quotidiance lectionis explosa, novum et nativum in divina scriptura dicendi genus, quale os dei locutum est, nosmet ipsi non sine quadam inauditi ac recentis nuper studii admiratione apprehendamus (Rudimenta, p. 1). 


\section{POUR L'HÉBREU OU CONTRE LES JUIFS ?}

La question du statut des juifs en Europe était d'une actualité brûlante en ces premières années du XVI ${ }^{\mathrm{e}}$ siècle. Ils avaient été expulsés d'Espagne en 1492 et la mort de Frédéric III, l'année suivante, les avait laissés sans défense en terre d'Empire. Les persécutions et expulsions se succédèrent tout au long du règne de son fils Maximilien (les expulsions de Styrie, Carinthie et Carniole furent signées dès 1494 , celle de Nuremberg quatre ans plus tard).

Enfin, le grand massacre de Lisbonne allait débuter moins d'un mois après la publication des Rudimenta, le 20 avril 1506. Ainsi, ce livre rédigé par un pieux catholique allemand appelant à étudier l'hébreu, occupe une place atypique au milieu de l'antijudaïsme dominant de son époque, d'autant que son auteur affirme si orgueilleusement qu'il va en tirer une gloire éternelle. Il allait effectivement en tirer une gloire durable, quoique l'opération ne fût pas un succès commercial à court terme, puisqu'en 1510, seuls 250 exemplaires sur 1000 en avaient été vendus.

Reuchlin était d'ailleurs très au fait de la question, puisqu'il avait lui-même publié l'année précédente un opuscule en allemand intitulé Pourquoi les juifs sont-ils depuis si longtemps dans le malheur ? ${ }^{10}$. Son argumentation y reprend trois lieux communs de la tradition chrétienne, qui ne brillent pas par leur originalité :

«En premier lieu, leur faute est beaucoup plus grande que celle pour laquelle ils ont été déportés à Babylone et pour laquelle ils sont restés 70 ans en exil. Ils doivent donc être punis beaucoup plus longtemps par Dieu. ${ }^{11}$ »

10. Doctor Johanns Reuchlins tütsch missiue, warumb die Juden so lang im ellend sind, imprimé à Pforzheim chez Thomas Anshelm. Ce texte a été réédité dans Johannes Reuchlin Sämtliche Werke, hsg. von Widu-Wolfgang Ehlers, Hans-Gert Roloff und Peter Schäfer, Frommann-Holzboog, StuttgartBad Cannstatt, (Band IV 1 : Schriften zum Bücherstreit), 1999, p. 1-12. C'est selon cette édition que je cite les extraits suivants.

11. Jtem zum ersten ist zugedenken das die sünd vnd übeltat dar vmb sye ytzund all $\beta$ gar lang vnd hart gestrafft werden vil großer sy dann einich andere mißtat, vnnd gar vil großer dann die darumb sye in Babel gefürtt sind gewesen, da sye allein lxx iar gefangen waren (op. cit., p. 5). 
«Ensuite, c'est tout le peuple juif qui est puni et non pas quelques-uns, car la faute a été commise par tout le peuple et par leurs descendants, aussi longtemps qu'ils demeureront juifs. ${ }^{12}$ »

«Enfin, leur faute est de n'avoir pas reconnu le messie dans Jésus, et de persister encore aujourd'hui dans leur aveuglement. ${ }^{13}$ »

En revanche, la singularité de Reuchlin se manifeste déjà, dans ce court texte, par le choix de ses sources. Ses autorités sont en effet exclusivement des sources hébraïques, qu'il cite soigneusement en hébreu : outre la Bible, ce sont Meister David Kimhi, Ribischlomo (Rachi), Ribimosche (Nahmanide), Der hochgelert meister Rabbi Mose, der Jud von Egiptten (Maïmonide) et la Kabbale Gematria. Enfin, il cite en araméen le Targum d'Onkelos. Il écrit donc ostensiblement comme si la Vulgate n'existait pas à ses yeux, ni les Pères de l'Église, ni les controversistes médiévaux, qui ne furent pourtant pas avares d'Adversus judceos. Cette posture, qui pourrait ne paraître qu'affectation d'érudition à un moderne, se situe délibérément à contre-courant de toute la tradition scolastique, pour laquelle il n'était pas concevable d'ignorer les maîtres chrétiens, surtout en matière de théologie. De plus, il conserva cette attitude dans ses publications ultérieures, comme le montrent la lecture de la lettrepréface des Rudimenta, autant que celle de l'Augenspiel.

Enfin, Reuchlin avait une conscience très claire de l'aggravation de la situation des juifs d'Europe, car il affirme paradoxalement que c'est elle qui l'a poussé à écrire ses Rudimenta, pour éviter que leur disparition n'entraîne celle de la langue divine.

«Car je me suis souvenu de la situation misérable des juifs de notre époque, qui ont été chassés de force pas seulement d'Espagne, mais aussi des frontières d'Allemagne et contraints de rechercher de nouveaux séjours jusque chez les Sarrasins, au point qu'il arrivera que la langue hébraïque viendra à manquer et à disparaître par notre faute,

12. Jtem zum andern [...] V $\beta$ dem allen zu mercken ist das die sünd darumb sye zerstrewt sind ein gemeine sünd de $\beta$ gantzen geschlechts syn ти $\beta$, darin all Juden verharren so lang sie iuden sind (ibid.).

13. Jtem zum dritten [...] Vnd disem allem nach so beschlüs ich das die Juden vmb kein andere sünd so lang zyt von got gestrafft werden dan alein vmb die gots lesterung die ire vordern an dem Messiah vnserm hern Jhesuh begangen haben vnd ire nachkommenden daryn verwilligt auch söllichs biß vff disen hütigen tag steet vnd angenem halten vnd haben (op. cit., p. 7). 
entraînant la grande ruine des saintes écritures. Alors, j'ai décidé de transmettre moi-même par ce livre l'expérience de la langue hébraïque aux chrétiens $[\ldots] .{ }^{14}$ »

\section{LA DÉGRADATION DES LETTRES SACRÉES}

Revenons au texte de la lettre-préface. Voici ses premiers mots :

«Très souvent, Denis, mon frère, j' ai réfléchi à la dégradation générale des Lettres sacrées qui, après avoir été provoquée, durant les années passées, par la multitude des sophismes, l'est maintenant surtout par l'étude de l'éloquence et le charme des poètes. Non seulement elles sont négligées, mais elles sont même considérées avec mépris par le plus grand nombre. ${ }^{15}$ »

Cette phrase introduit une problématique complexe. En premier lieu, la dénonciation de la « dégradation des Lettres sacrées » renvoie en écho à la « dégradation des bonnes Lettres », thème ressassé par les humanistes depuis Pétrarque. Leur revendication du retour à l'élégance de la langue latine, corrompue par un millénaire de barbarie, visait en effet essentiellement les lettres profanes, tombées dans l'oubli et redécouvertes par leurs propres efforts.

Ils évitaient en revanche d'aborder le domaine des Lettres sacrées (Ancien et Nouveau Testament), et ce pour plusieurs raisons. En premier lieu, les textes bibliques ne les intéressaient guère pour leurs qualités littéraires et ne pouvaient rivaliser avec la «meilleure littérature » de Cicéron, Virgile ou Homère. Mais, pour ceux d'entre d'eux que la religion préoccupait, une autre raison freinait leur curiosité : l'étude de la Bible était soumise à l'autorité et à la surveillance des facultés de théologie, qui veillaient jalousement à cette prérogative et voyaient d'un très mauvais œil les immixtions

14. Sane recordatus miseros nostra atate Judceorum casus, qui non tam ex Hispanice, quam etiam Germanice nostra finibus pulsi coguntur alias sibi sedes quacere atque ad Agarenos divertere, quo futurum est, ut tandem hebraica lingua cum sacrarum literarum magna pernicie penes nos posset desinere atque evanescere, - mecum ipse constitui ad tradendam Christianis hebraici sermonis peritiam hoc libro [...] (Rudimenta, p. 3).

15. Persape mihi cogitanti de communi sacrarum literarum jactura, Dionysi frater, qua cum multitudine sophismatum annis superioribus, tum maxime nunc propter eloquentice studium et pæetarum amænitatem non modo negliguntur, verum etiam a quam plurimis contemptui habentur (Rudimenta, p. 1). 
possibles des philologues humanistes sur leur terrain. Un laïc qui s'aventurait publiquement dans ce domaine s'exposait aux poursuites de l'Inquisition.

Dès le milieu du XIII ${ }^{\mathrm{e}}$ siècle, le franciscain Roger Bacon avait lancé une attaque en règle contre la dégradation de la Vulgate et réclamé que les théologiens étudient la Bible en hébreu et en grec, mais il n'avait pas été entendu. Il écrivait par exemple dans le chapitre IV de son Opus majus (dont la Pars tertia est intitulée De utilitate grammatica) :

«La sixième raison concerne la correction des erreurs et des fautes innombrables dans le texte de la théologie autant que de la philosophie, et pas seulement dans la lettre, mais aussi dans le sens. Et je justifie la nécessité de cette correction par l'étendue de la corruption [des textes]. Et puisqu'il est plus grave et dangereux de se tromper dans le texte de Dieu que dans un texte philosophique, j'appliquerai donc la compétence des langues à la correction du texte sacré, dont la nécessité est patente au vu de la corruption infinie de l'édition de la Vulgate qu'on nomme parisienne..$^{16} »$

La Vulgate «parisienne» était celle que l'université de Paris avait essayé d'unifier depuis près d'un siècle pour l'usage de ses enseignements de théologie, mais sans grand succès comme l'atteste Bacon. Par la suite, les luttes d'influence entre les ordres prêcheurs dominicains et franciscains, et par leurs écoles respectives albertistes, thomistes, scotistes puis occamistes, n'avaient pas amélioré la situation. Chacun défendait les corrections de ses scriptoria et cherchait à imposer ses dernières éditions.

Au final, les rugosités et les incohérences du texte biblique étaient attribuées aux maladresses des copistes - de l'ordre rival bien sûr -, voire à la «naïveté » de ses auteurs (Moïse pour l'Ancien Testament et les Apôtres pour le Nouveau). Cependant, la

16. Sexta ratio est propter correctionem errorum et falsitatum infinitarum in textu tam theologice quam philosophice, non solum in litera sed in sensu. Quod autem correctio sit necessaria probo per corruptionis magnitudinem. Et quoniam violentius et periculosius erratur in textu Dei quam in textu philosophice, ideo convertam linguarum potestatem ad corruptionem textus sacri, ut pateat necessitas earum propter corruptionem infinitam exemplaris vulgati quod est Parisiense (Nolan \& Hirsch, The Greek Grammar of Roger Bacon, Cambridge University Press, 1902, p. 94). 
règle restait chez les clercs d'affecter de croire que la Vulgate était parfaite, alors même qu'aucun lettré sérieux ne pouvait contester qu'elle nécessitât une révision complète.

L'observation initiale de Reuchlin à son frère sur la dégradation des Lettres sacrées n'a donc rien pour surprendre de la part d'un humaniste. Ce qui est en revanche plus étonnant, c'est son commentaire sur ses causes. Il en distingue en effet deux, l'une ancienne et l'autre récente.

\section{LES SOPHISTES ET LES POÈTES}

La première cause alléguée n'est pas l'ignorance des copistes, mais la multitude des «sophismes »-allusion transparente à la scolastique. Le terme «sophisme » désigne de façon directe la débauche de débats contradictoires (notamment quodlibétiques) qui avaient envahi les facultés des arts. Mais Reuchlin vise sans doute plutôt ici l'impérialisme de la dialectique dans l'enseignement de théologie, et en particulier le commentaire obligatoire des Livres des Sentences de Pierre Lombard, dont l'étude avait quasiment remplacé celle du texte biblique. En outre, cette pratique imposait une confusion textuelle supplémentaire car elle imposait que les leçons de la Vulgate utilisée correspondissent avec celles de Pierre Lombard, qui avait écrit selon la Parisienne de son temps (au milieu du $\mathrm{XII}^{\mathrm{e}}$ siècle).

Cette critique n'était pas non plus nouvelle. Roger Bacon, encore lui, s'était déjà plaint amèrement du mépris dans lequel était tenue la lecture de la Bible dans les facultés de théologie. Voici ce qu'il en disait :

« Il y a en vérité sept péchés dans l'étude principale, qui est celle de la théologie :

- Le premier est que la philosophie a dans les faits la préséance sur la théologie. $[\ldots]^{17}$

17. Sunt vero septem peccata studii principalis quod est theologia ; unum est quod philosophia dominatur in usu theologiam (Opus minus, édité dans Fr. Rogeri Bacon opera quadam hactenus inedita, ed. J. S. Brewer, Londres, Longman et Green, 1859, p. 322). 
- Le second péché est que les théologiens ne pratiquent pas les meilleures sciences qui touchent le plus à la théologie. $[\ldots]^{18}$

- Le troisième péché est que les théologiens ne pratiquent pas ces quatre sciences qui sont la base de la théologie. [... ${ }^{19}$

- Le quatrième péché est que la prééminence est donnée à une seule sentence magistrale du texte de la faculté de théologie, à savoir le Livre des Sentences. $[\ldots]^{20} »$

«En effet, celui qui fait le cours des Sentences dispose de la meilleure heure de cours selon ses désirs. On lui donne un assistant et une chambre chez les moines. Par contre, celui qui fait le cours de la Bible n'y a pas droit et il doit mendier son heure de cours, selon le bon vouloir du professeur des Sentences. [...] $]^{21}$

- Le cinquième péché est plus grave que tous les précédents. En effet, le texte de la Vulgate que l'on appelle parisienne est pour sa majeure partie horriblement corrompu. $[\ldots]^{22} \gg$

Retour à la Bible, donc, mais dans quel texte ? Si l'on excepte quelques tentatives isolées, comme celle de la Superscriptio lincolnensis promue par Robert Grosseteste à la même époque ${ }^{23}$, les corrections de la Bible latine ne parvinrent jamais, au cours du Moyen Âge, à excéder les remises à jour des diverses gloses qui l'accompagnaient. La raison principale en était que les deux « autres » langues sacrées, le grec et l'hébreu, n'étaient enseignées dans aucune université, si bien que les correcteurs devaient nécessairement être des conversos instruits ou se faire aider par des juifs, courant ainsi le risque évident de se faire accuser d'hétérodoxie. Les gloses

18. Secundum peccatum est quod scientice optima maxime contingentes theologia non sunt in usu theologorum (ibid., p. 323).

19. Tertium peccatum est quod illae scientice quatuor quod sunt in usu theologorum, sunt ab eis ignotae. (ibid., p. 325).

20. De Pierre Lombard.

21. Quartum peccatum est quod prafertur una sententia magistralis textui facultatis theologica, scilicet liber sententiarum [...] Nam ille qui legit Sententias habet principalem horam legendi secundum suam voluntatem, habet et socium et cameram apud religiosos. Sed qui legit Bibliam, caret his et mendicat horam legendi, secundum quod placet lectori Sententiarum (op. cit., p. 328-329).

22. Quintum peccatum est majus omnibus pradictis. Nam textus est pro majori parte corruptus horribiliter in exemplari Vulgato, hoc est Parisiensi (ibid., p. 330).

23. Voir à ce sujet The Cambridge History of the Bible, vol. 2, ed. G. W .H. Lampe, Cambridge University Press, 1969, p. 152 sqq. 
les plus fameuses furent rédigées au XIV siècle par l'hébraïsant Nicolas de Lyre ${ }^{24}$, s'inspirant principalement des commentaires de Rachi. Leur succès ne se démentit jamais, comme l'atteste Reuchlin (Rudimenta, p. 547-548), malgré les vigoureuses critiques dont elles furent l'objet, un siècle plus tard, de la part de Paul de Burgos ${ }^{25}$, lui-même contre-attaqué par Matthias Döring ${ }^{26}$. La plupart des grandes Bibles incunables in-folio présentaient ainsi le texte de la Vulgate entouré par l'ensemble des gloses, c'est-à-dire les Explicationes de Guillaume Brito, les Postilla de Nicolas de Lyre, les Additiones de Paul de Burgos et les Replicationes de Matthias Döring.

Il n'y a pas lieu de s'attarder sur cette polémique entre glossateurs ni sur leurs qualités respectives. On se contentera de noter que Nicolas de Lyre était franciscain et Paul de Burgos, dominicain. Il n'est donc pas vraiment étonnant que le franciscain Matthias Döring ait pris la défense de son confrère et critiqué Paul de Burgos dans des termes particulièrement violents.

C'est seulement au milieu du XV' siècle, alors que cette controverse faisait rage, que quelques humanistes commencèrent à appliquer la critique philologique aux textes sacrés grecs - sans entamer pour autant le monopole des théologiens scolastiques. Aidé par le cardinal Bessarion, Lorenzo Valla publia sa Collatio Novi Testamenti, mais l'ouvrage ne sortit pas des cercles romains. Il fut imprimé en 1505 par Érasme à Paris ${ }^{27}$, soit près de cinquante ans après la mort de son auteur. En 1506, les humanistes qui s'intéressaient à la question

24. Postilla perpetuce in Vetus et Novum Testamentum. Edward A. Gosselin a répertorié pas moins d'une bonne centaine d'éditions de ces postilles jusqu'aux années 1520 ( A Listing of the Printed Editions of Nicolaus of Lyra », Traditio, vol. XXVI, New York, 1970).

25. Au nombre de plus d'un millier, elles étaient généralement intitulées Additiones ad Postillam magistri Nicolai de Lyra.

26. Au nombre de quatre cents, elles étaient généralement intitulées Replica ou Defensorium postillae Nicolai Lyrani.

27. Chez Josse Bade, sous le titre Laurentii Vallensis... in latinam Novi Testamenti interpretationem ex collatione Gracorum exemplarium Adnotationes apprime utiles. 
de l'hébreu, généralement sous l'influence de Pic de la Mirandole ${ }^{28}$, se comptaient encore sur les doigts de la main.

Après une attaque aussi frontale contre les scolastiques, le lecteur s'attend à ce que Reuchlin se range sous l'étendard de leurs ennemis jurés, à savoir les «poètes », en d'autres termes les humanistes - surtout après avoir lu les vers d'Horace de la vraie-fausse première page. Or, non seulement il n'en fait rien, mais il dénonce au contraire les poètes comme causes de la dégradation récente des Lettres sacrées, reprenant à son compte la vieille critique d'impiété que les théologiens adressaient déjà à Boccace. Détail piquant, c'est par l'image fortement poétique d'Ulysse résistant aux chants des Sirènes (qui attirent le chrétien vers l'Enfer par la beauté de leurs chants) qu'il illustre sa charge contre les poètes :

«Il m'est enfin venu à l'esprit un remède approprié pour que les écritures de la sainte Bible ne finissent par disparaître et pour que, de la même façon, le cheminement de nos âmes ne s'abîme pas dans les Enfers, avec le doux chant des Sirènes, qu'Ulysse n'a même pas écouté. ${ }^{29}$ »

Il n'est d'ailleurs pas besoin de pousser beaucoup la métaphore pour deviner Reuchlin lui-même sous les traits du nouvel Ulysse, capable d'écouter les Sirènes, tout en résistant à leurs mélodies captieuses. On le devine même à un double titre parce qu'il était le seul Allemand de son temps connaissant le grec, donc capable d'apprécier le chant d'Homère - et de ses Sirènes. Sa connaissance du grec était en effet un de ses principaux titres de gloire, comme il le rappelle largement dans sa lettre-préface. On citera à ce propos l'anecdote célèbre, racontée par son petit-neveu Philipp Melanchthon, selon laquelle, lors d'un de ses voyages à Rome (sans doute en 1490), il aurait rencontré Jean Argyropoulos en train de faire une leçon sur Thucydide. Le savant grec ayant présenté son livre à

28. On se référera à François Secret, Les Kabbalistes chrétiens de la Renaissance, Milan, Archè, 1985 et à Louis Valcke, Pic de la Mirandole, un itinéraire philosophique, Paris, Les Belles Lettres, 2005.

29. In mentem venit tandem oportuni cujusdam remedii, ne sancta biblice scriptura vel aliquando tota pereat et simul animarum nostrarum progressus cum suavi cantu Sirenum, quas ne Ulysses quidem audiret, ad inferos abeat (Rudimenta, p. 1). 
l'étranger de passage, ce dernier aurait traduit (en latin et «à livre ouvert ») cet auteur particulièrement difficile. Très impressionné, Argyropoulos aurait alors déclaré : «Notre Grèce, dans notre exil, a survolé les Alpes. ${ }^{30} \gg$ Le goût de Reuchlin pour le grec était d'ailleurs si poussé qu'il traduisit son nom (Räuchlein : «petite fumée ») en grec : Capnion, comme le ferait après lui Philipp Melanchthon, dont le nom allemand était Schwarzerd.

\section{LE DÉGOÛT DES TRADUCTIONS}

Pour les hommes du $\mathrm{Xx}^{\mathrm{e}}$ siècle, la revendication du retour à l'hébreu biblique paraît banale, tant nous sommes accoutumés à la dialectique éternellement renouvelée entre les textes-sources et leurs traductions. Il en allait tout autrement dans les premières années $\mathrm{du} \mathrm{XVI}^{\mathrm{e}}$ siècle, dans une Europe dont le latin légitimait encore toutes les institutions. Cette légitimation s'appuyait elle-même sur celle de l'Église catholique, dont le clergé n'utilisait aucune autre langue dans sa liturgie. En d'autres termes, dans l'Europe catholique, la langue de la Bible était le latin. Elle avait bien sûr déjà été traduite en diverses langues vernaculaires, mais uniquement pour l'usage du peuple et pour l'évangélisation des païens ou des illettrés. Pour les clercs, l'usage d'une autre langue que le latin était proprement inimaginable, puisqu'il était précisément le signe de leur appartenance à cet ordre privilégié. D'ailleurs, les auteurs de traductions en vernaculaire étaient généralement soupçonnés (à juste titre) de chercher à battre en brèche le pouvoir du clergé - que l'on pense à Wycliff ou à Luther.

Au moment où Reuchlin écrit cette lettre, c'est en latin que Luther enseigne depuis deux ans la théologie à la toute nouvelle université de Wittenberg. Il y commente scrupuleusement les Sentences de Pierre Lombard et ne lit la Bible que selon la Vulgate, tout comme ses confrères de Cologne ou de Paris. Quand Reuchlin

30. «Græcia nostra nostro exilio transvolavit Alpes. » (Declamatio de Capnio Phorcensis, 1552, reproduite dans Melanchthonis Opera Omnia, Bretschneider, 1843, vol. XI, col. 1005 sq.) 
parle de son dégoût pour les traductions de la Bible, c'est donc de la Vulgate qu'il parle, et son évocation du proverbe vilescit quotidianum est pour le moins provocatrice (sans parler du non moins péjoratif fastidium) :

« J'ai donc réfléchi à la façon de remédier à ce danger. Avant tout, étant donné que le désir d'une grande variété (j'ignore d'où cela provient) se trouve greffé dans nos âmes, au point d'évoquer ce proverbe : "Ce qui est quotidien se déprécie". ${ }^{31}$ »

Or, si les humanistes mettaient leur point d'honneur à restaurer les textes anciens selon la critique philologique la plus poussée, ils militaient également pour l'élégance des traductions latines. Cette position, soutenue dès les premières années du Xve siècle par Manuel Chrysoloras (le premier professeur de grec à Florence), fut notamment à l'origine la polémique qui opposa son élève Leonardo Bruni à Ambrogio Traversari et à Niccolò Niccoli à propos des traducteurs scolastiques de grec. On se rappellera la charge lancée par Bruni contre Robert Grosseteste, à propos de sa traduction (vers 1235) de l'Éthique à Nicomaque, dans De interpretatione recta: «Qu'est-ce que je lui reproche donc ? Uniquement son ignorance des Lettres ! ${ }^{32}{ }$

Encore ne s'agissait-il que d'une traduction d'Aristote, texte profane s'il en fut. Quand la critique textuelle se tourna vers les textes bibliques, elle se heurta à une résistance acharnée de la part de l'Église catholique, en vertu du principe de l' « authenticité » de la Vulgate dont l'auteur, saint Jérôme, avait été inspiré par le SaintEsprit. On rappellera que l'interdiction de la lecture de la Bible dans les langues-sources (le grec et l'hébreu), promulguée un siècle plus tard lors de la IVe session du concile de Trente, en 1546, ne serait officiellement levée que par l'encyclique Divino afflante spiritu de Pie XII en 1943.

31. Ad istum autem modum ei periculo succurrendum putavi. Primum omnium, cum sit in animis nostris (nescio unde) insitum multa varietatis desiderium, quo fit ut versetur in ore ille adagium: vilescit quotidianum (Rudimenta, p. 1).

32. «Quid igitur in illo reprehendi ? Imperitiam solummodo litterarum. » (Leonardo Bruni, De interpretatione recta, dans Bruni Leonardo, Opere letterari e politiche, a cura di Paolo Viti, Torino, Unione Tipografico-Editrice Torinese, 1996, p. 152.) 
Reuchlin s'aventurait donc sur un terrain miné, tout comme allait le faire Érasme quelques années plus tard, car l'approche «humaniste » de la Bible remettait en question de proche en proche l'ensemble de l'édifice de la théologie scolastique, donc de l'autorité de l'Église romaine.

\section{L'AUTRE VOIE}

Deux voies s'offrent donc au savant: la première consiste à corriger la Vulgate, à partir des meilleurs manuscrits et de leurs leçons les plus anciennes, voire à mettre en chantier une nouvelle traduction de la Bible. Cette voie, explorée par Lorenzo Valla, fut poursuivie par Érasme et aboutit à la publication de sa nouvelle traduction du Nouveau Testament en février 1516 sous le titre de Novum Instrumentum, provoquant le scandale que l'on sait ${ }^{33}$. Reuchlin aurait pu s'y engager lui aussi et se lancer dans l'entreprise d'une nouvelle traduction de l'Ancien Testament de l'hébreu en latin, tout en s'appuyant sur la Septante qu'il pouvait lire en grec. Mais il ne le fit pas et c'est seulement en 1528 que Sante Pagninus publia à Lyon un Ancien Testament retraduit en latin à partir de l'hébreu.

Reuchlin, arguant de son dégoût pour les traductions, plaide pour une autre voie, c'est-à-dire la lecture du texte original. Il ne nous éclaire pas sur les causes profondes de ce dégoût, mais peutêtre s'explique-t-il simplement par le fait que l'outil permettant la compréhension ultime de son objet - la Kabbale, permettant d'accéder au sens caché de la Bible - n'est par définition accessible que dans sa langue originale, c'est-à-dire l'hébreu. La Kabbale est hébraïque dans son contenu-même et pas seulement dans sa forme. Elle ne peut s'approcher en latin, ni a fortiori dans aucune langue vernaculaire. L'hébreu de la Bible étant la langue «naïve» de Dieu, il faudra donc venir aux mots mêmes de l'hébreu et à leurs

33. Voir à ce sujet Jean-Christophe Saladin, La Bataille du grec à la Renaissance, Paris, Les Belles Lettres, 1999, passim. 
lettres. En un mot, il faut retourner à l'école et étudier les rudiments de cette langue.

Reuchlin tourne ainsi le dos à ses collègues humanistes, en affirmant - à la suite de Pic de la Mirandole et de ses amis juifs que la vérité ne se trouve pas dans le latin, mais dans l'hébreu. Il se démarque encore davantage des théologiens scolastiques, en récusant implicitement le principe des quatre sens de l'Écriture (dont les exégèses pouvaient se pratiquer en latin), pour lui préférer la Kabbale. Il leur reproche enfin de s'alimenter à une source triplement fausse : le commentaire d'une Vulgate latine dégradée, en lieu et place du texte hébreu de la Bible.

L'hébreu n'est alors plus considéré comme une langue dont le statut est comparable à une autre, même par le biais de sa traduction éventuelle. En tant que parole originelle du Dieu créateur, il est la « source d'où jaillit toute la théologie » (a quo fonte omnis theologia scaturiuit) et se voit alors désigné par une métaphore très chargée affectivement, puisqu'il faut «l'embrasser à pleins bras et le couvrir de baisers autant que nos petits bébés ${ }^{34}$.

Le propos de l'auteur se précise : convaincre ses contemporains de se mettre à l'étude de l'hébreu. Pour y parvenir, il faut remplir cependant trois conditions : trouver des textes, des professeurs et des élèves.

\section{UN CURSUS INVERSÉ}

La première difficulté est déjà résolue : on trouve, dit Reuchlin, des Bibles en hébreu à bas prix en Italie : « Or, voilà qu' on imprime des Bibles en hébreu un peu partout en Italie, que chacun peut facilement acheter à bas prix s'il le désire. ${ }^{35}$ »

34. Non aliter, quam liberi nostri infantes exosculanda, et totis brachiis amplexanda (Rudimenta, p. 1).

35. Cum igitur hebraica biblice passim in Italia imprimantur, quas sibi quisque pauco are mercari facile queat (Rudimenta, p. 1). 
Sans doute fait-il allusion à l'édition in-folio publiée en 1488 par l'imprimeur Joshua Salomon Soncino. Il s'en était procuré un exemplaire pour six ducats d'or lors de son séjour à Rome en 1492. Son admiration devant la modicité du prix s'explique peut-être par le fait que l'empereur lui avait fait cadeau d'une Bible juive manuscrite (Codex reuchlinianus) qui avait coûté la bagatelle de 300 ducats - soit un an et demi de son salaire annuel de juge « triumvir» de la Ligue souabe.

Cette première difficulté levée, reste à trouver des professeurs d'hébreu, capables « d'exposer la langue hébraïque aux chrétiens à la façon des grammairiens ${ }^{36}$. Le terme n'est pas innocent. Il s'agit bien du grammaticus, c'est-à-dire du maître d'école primaire, connu depuis l'antiquité grecque pour enseigner aux enfants les rudiments de la littérature. Existe-t-il des hommes ayant cette compétence pour l'hébreu? Peut-être, mais aucun ne s'est manifesté. Qui étaient ces personnages rares? Sans doute des théologiens, mais ils ont jugé trop fatigant ou « indigne de leur rang » de reprendre des fonctions aussi humbles après avoir exercé leurs talents grassement rémunérés dans les plus hautes sphères universitaires.

Faute de candidats, c'est donc Reuchlin qui, le premier, se lance dans cette aventure héroïque mais assurément peu lucrative. Rappelons que le cursus des études depuis l'Antiquité commençait par la grammaire, suivie par la rhétorique, et couronnée par la philosophie. Cet ordre était resté celui des trois matières étudiées lors du trivium dans les facultés des arts médiévales. Il fournissait aux enseignants une hiérarchie dans laquelle le pauvre grammaticus était proverbialement connu pour ses revenus misérables, tandis que ses confrères rhéteurs ou philosophes dispensaient leurs cours à prix d'or. Devenir grammaticus était donc une régression sociale caractérisée. Reuchlin va s'employer à démontrer qu'il n'en est rien.

Il cite fièrement comme modèles trois célèbres Latins qui, dit-il, ont précisément parcouru ce cursus inversé en commençant par

36. Linguam hebraicam grammaticorum more christianis demonstraret (Rudimenta, p. 1). 
enseigner la philosophie, puis la rhétorique, et enfin la grammaire. Il tient leur histoire du De grammaticis et rhetoribus de Suétone ${ }^{37}$.

\section{L'ÉPICURIEN, LE PHILOLOGUe ET LA SiRÈne}

Le premier est Aurelius Opilius, à propos duquel Reuchlin reproduit presque mot à mot la notice de Suétone : « Aurelius Opilius, affranchi d'un épicurien, enseigna d'abord la philosophie, puis la rhétorique et finalement la grammaire. ${ }^{38}$ » On notera que l'homme est présenté comme affranchi d'un épicurien, détail que Reuchlin n'a pas jugé bon de répéter, cette qualité n'étant assurément pas la meilleure référence pour aborder les Lettres sacrées. Il est vrai que seul le maitre était épicurien, mais il faut penser que cette particularité avait peut-être contaminé l'esclave, sans quoi Suétone ne l'aurait pas mentionnée.

Le second est le «philologue Atéius Pretextatus ». La notice de Suétone précise qu'il était réputé « rhéteur parmi les grammairiens et grammairien parmi les rhéteurs ${ }^{39}$, et qu'il « s'était lui-même qualifié de philologue ${ }^{40}$. On y lit un peu plus loin : «Il semble qu'il ait pris le nom de philologue parce que, tout comme Ératosthène, qui fut le premier à revendiquer ce surnom, il était réputé pour ses connaissances nombreuses et variées. ${ }^{41}{ }^{~}$ C'est un modèle idéal pour un humaniste aussi polyvalent que Reuchlin.

Le troisième est Valerius Caton, «que l'on nommait la Sirène latine ». Cette formule est également empruntée à la notice de

37. Cet ouvrage paradigmatique de l'humanisme, dont le manuscrit incomplet avait été retrouvé au monastère de Hersfeld grâce à l'opiniâtreté du Pogge et de Niccolò Niccoli, avait été publié vers 1574 à Venise puis par Philippe Beroald à Bologne en 1504.

38. Aurelius Opilius Epicurei cuiusdam libertus philosophiam primo, deinde rhetoricam, nouissime grammaticam docuit. (Suétone, De grammaticis... VI, 1).

39. inter grammaticos rhetorem, inter rhetores grammaticum (Ibid. X, 1).

40. philologus ab semet nominatus (Ibid. X, 2).

41. Philologi appellationem assumpsisse uidetur quia sicut Eratosthene, qui primus hoc cognomen sibi uindicauit, multiplici variaque doctrina censebatur (Ibid. X, 4). 
Suétone, citant un distique qui circulait à Rome au sujet de ce personnage :

«Caton, le grammairien, Sirène latine,

Seul à enseigner et à fabriquer les poètes. ${ }^{42}$ »

Suétone précise qu'il «enseigna à de nombreux élèves nobles, et fut reconnu comme un professeur particulièrement excellent pour ceux qui se destinaient à la poésie ${ }^{43}$. Comparer un poète - voire un professeur de poésie - à une Sirène n'a rien de très original. On observera cependant que c'est la seconde fois que ces êtres mythologiques apparaissent dans la lettre. Or cette fois, c'est Reuchlin lui-même qui s'attribue le masque de la Sirène par un jeu d'identification redoublée (je voudrais ressembler à Valerius Caton que l'on surnommait la Sirène latine). Son goût immodéré pour la poésie lui fait ainsi réhabiliter au passage (peut-être involontairement) les malheureuses Sirènes qu'il avait si durement condamnées dès ses premiers mots.

\section{LE PHILOSOPHE ET LE RHÉTEUR}

Reuchlin peut ainsi se proclamer grammairien en se réclamant du patronage de personnages célèbres et authentifiés par l'autorité littéraire (quoique païenne) de Suétone. Cependant, il lui faut encore répondre par avance à ses détracteurs, qui ne manqueront pas de lui reprocher son manque de compétence dans les deux premiers domaines considérés, à savoir la philosophie et la rhétorique.

En ce qui concerne la philosophie, il a beau jeu de citer son Capnion vel de verbo mirifico ${ }^{44}$, dans lequel il a exposé sa première synthèse d'inspiration kabbalistique. L'ouvrage, publié en 1494, est

42. Cato grammaticus, Latina Siren, / qui solus legit ac facit poetas... (Ibid. XI, 2).

43. docuit multos et nobiles uisusque est peridoneus praceptor maxime ad poeticam tendentibus (Ibid. XI, 2).

44. Cet ouvrage a été réédité et traduit en allemand dans le premier volume des Johannes Reuchlin Sämtliche Werke (Band I, 1), FrommannHolzboog, Stuttgart, 1996. 
un gros dialogue philosophique mettant en scène trois personnages : le philosophe Sidonius «anciennement épicurien, mais qui ne se soumet plus à aucune doctrine et actuellement ouvert à toute proposition ${ }^{45}$, le kabbaliste juif Baruchias et Reuchlin lui-même, sous son nom grec de Capnion. Il y exposait sous son nom la théorie de la transformation du tétragramme juif «IHUH» (le nom de Dieu) en pentagramme chrétien «IHSUH » (le nom de Jésus). Cette théorie s'appuyait notamment sur la $14^{\mathrm{e}}$ conclusion cabalistique de Pic de la Mirandole, concernant « la lettre scin qui occupe la place centrale dans le nom de Jésus ${ }^{46}$.

S'étant incontestablement posé en philosophe hébraïsant, Reuchlin peut annoncer son œuvre actuelle de grammairien de l'hébreu. Mais, pour que son cursus soit complet, il lui manque le chaînon de la rhétorique. Or, il ne mentionne pas d'ouvrage de rhétorique hébraïque de sa composition, pour la bonne raison qu'il n'en a pas écrit. Sa démonstration serait donc incomplète, sauf à se référer à un curieux petit livre, intitulé Liber congestorum de arte predicandi ( «Livre de tout ce qui a été rassemblé sur l'art de la prédication») qu'il avait publié deux ans plus tôt. Il s'agit d'un traité de rhétorique non pas hébraïque mais latine, adressé à un couvent de dominicains. Cet ouvrage est généralement tenu pour secondaire par rapport au reste des œuvres de Reuchlin, mais il me semble éclairant d'en faire une brève présentation car son contenu est fort inattendu.

Les traités sur l'art de faire les sermons (ars predicandi) étaient innombrables à la fin du Moyen Âge. Ils présentaient généralement des recueils d'exempla ou de citations de l'Écriture à utiliser selon les périodes du calendrier liturgique. Celui de Reuchlin n'a rien à voir avec eux : c'est un pur traité de rhétorique païenne, directement décalqué des Divisions de l'art oratoire et des Topiques de Cicéron. On en jugera par le sommaire qu'il en donne au début de l'ouvrage :

45. Sidonius de schola Epicuri primum putatus, deinde inventus in nullius verba iurasse, omnifariam philosophus (op. cit., préface, p. 10).

46. Per literam scin, qua mediat in nomine Iesu (cf. Louis Valcke, Pic de la Mirandole, Paris, Les Belles Lettres, 2005, p. 190). 
«L'art de la prédication comprend trois parties : l'invention, la mémoire et la prononciation, que nous réalisons facilement par la nature, les règles, l'imitation et l'entraînement. ${ }^{47}$ »

La première partie, intitulée De inventione, commence par ces mots :

«Le discours (sermo) est constitué par l'introduction (principium), la lecture (lectio), la division, la confirmation (confirmatio), la réfutation et la conclusion (conclusio). L'introduction se concilie l'auditeur, la conclusion l'émeut, les autres parties l'instruisent, toutes le réjouissent. ${ }^{48} »$

Cicéron, dans les Divisions de l'art oratoire, avait écrit :

«Il y a quatre parties dans le discours. La première et la dernière doivent émouvoir l'âme, à savoir l'introduction (initium) et la péroraison (peroratio). La seconde (narratio) et la troisième, confirmation (confirmatio), rendent le discours convaincant. ${ }^{49}{ }^{»}$

Reuchlin s'est contenté de remplacer la seconde partie du discours (la narration) par la lecture (en l'occurrence d'un texte sacré), ce qui lui permet d'appliquer les règles cicéroniennes de composition d'une plaidoirie judiciaire à la prédication. Il fera ensuite appel à toutes les techniques d'argumentation classique : après avoir lu le texte, le prédicateur en présentera les parties (divisiones), puis il argumentera sur les thèmes repérés (confirmatio) et réfutera ses adversaires (refutatio).

La seconde partie (De memoria) présente la technique des loci communes. Elle commence par un rappel de leur définition traditionnelle :

«La mémoire fabriquée est donc constituée d'images formées dans les lieux, de la même façon que l'écriture de la tablette est constituée de

47. Artis pradicandi partes sunt tres, inuentio, memoria, \& pronunciatio, quas natura, prceceptis, imitatione, \& exercitatione facilè consequemur (Liber congestorum, $\left.\mathrm{f}^{\circ} \mathrm{A} . \mathrm{iij}\right)$. Je cite cet ouvrage selon l'édition anglaise Londini, per Henricum Synneman, anno 1570, accessible sur le site Gallica de la BNF.

48. Sermo constat Principio, lectione, diuisione, confirmatione, confutatione, conclusione. Principium conciliat auditorem, conclusio mouet, catera partes docent, universa delectant (Ibid.).

49. Quattuor[orationis] partis esse, quarum prima et postrema ad motum animi ualet ; is enim est initiis et perorationibus concitandus. Secunda, narratio et tertia, confirmatio, fidem facit orationi (Cicéron, Partitiones oratoriae, VIII, 27). 
lettres formées dans la cire. [...] Ainsi, les images, comme les lettres, disparaissent du lieu où nous ne les utilisons pas. En revanche, les lieux, comme la cire, persisteront. ${ }^{50}{ }^{»}$

Voici comment Cicéron les définissait :

«Dans l'écriture, on distingue entre les lettres et ce sur quoi elles sont inscrites, de même le travail de la mémoire utilise les lieux à la façon de la cire et y place les images comme des lettres. ${ }^{51}$ »

La troisième partie du traité donne des conseils de bon sens sur l'élocution et les postures de l'orateur. Reuchlin offre ainsi à ses amis religieux une méthode de prédication dans la solide tradition de la rhétorique païenne et son Liber congestorum peut alors être considéré comme le chaînon manquant de sa «carrière inversée » - mais il se garde de le mentionner.

\section{HoMo TRILINGUIS}

Après avoir légitimé son choix de devenir grammairien, Reuchlin s'attarde maintenant à justifier la qualité de sa formation personnelle dans les «trois langues ». Par une démonstration très cicéronienne, il en prend pour prétexte la réfutation de critiques supposées selon lesquelles sa carrière de magistrat ne lui laisserait pas les loisirs nécessaires pour s'adonner aux Lettres - il exerçait en effet les fonctions de juge de la Ligue souabe depuis 1502 (triumvir car ces juges étaient trois).

Son argumentation va consister à prouver par comparaison qu'il a réussi, tout au long de sa vie, à pratiquer en même temps les études et l'enseignement, tant par des cours que par des publications, dans chacune des branches qu'il a abordées.

50. Constat igitur artificialis memoria ex locis, \& imaginibus, sicut tabula scriptura ex cera, \& literis. [...] Nam imagines, sicut litera, delentur ubi nihil utimur. Loca verò, sicut cera remanebunt (Reuchlin, Liber congestorum, non paginé).

51. Nam ut illa constat ex notis litterarum et ex eo in quo imprimuntur illa nota, sic confectio memoria, tamquam cera, locis utitur et in his imagines ut litteras conlocat (Cicéron, Partitiones oratorice, VIII, 26). 
Pour les Lettres latines, il a achevé son trivium à Paris (Priscien de Césarée), qu'il a ensuite transmis au public sous la forme de son dictionnaire latin (Breviloquus Vocabularius) à Bâle.

Pour les Lettres grecques, il en a étudié les rudiments auprès d'Andronicos Contoblacas à Bâle, puis les a enseignées à Orléans et à Poitiers. Il a également publié un manuel de grec (Micropcedia). Il reviendra plus en détail sur la suite de ces études (Rudimenta, p. 547-548).

Il a étudié le droit civil à Orléans, dont il a obtenu le baccalauréat en 1479, puis la licence à Poitiers. Il l'a enseigné dans cette ville, puis à Tübingen. Cela lui donne l'occasion de balancer les deux termes de la jolie période : «C'est ainsi que j'ai étudié le droit des Latins tout en enseignant les préceptes des Grecs. »

Pour l'hébreu enfin, il a étudié à Linz auprès de Jacob Iehiel Lohans ${ }^{52}$, puis en Italie, auprès d'Abdia Sforno ${ }^{53}$.

Le présent ouvrage constitue donc une belle entrée en matière à son programme d'enseignement des Lettres hébraïques et il peut proclamer en fanfare qu'il est, à ce jour, le premier à avoir accompli les vœux du pape Clément V, inspiré par Raymond Lulle, qui recommandait aux quatre universités de Bologne, Paris, Salamanque et Oxford de créer des chaires pour l'enseignement de l'hébreu, de l'arabe, du syriaque et du grec. Ce décret (Inter sollicitudines) était resté un vœu pieux car aucune université ne voulut financer un souhait aussi incongru à cette époque. Reuchlin peut donc d'ores et déjà annoncer ses prochains ouvrages (Rudimenta, p. 4). Nous savons que ce programme fut rempli puisqu'il publia par la suite le De accentibus et surtout le De arte cabalistica. Au final, c'est aux Lettres hébraïques que l'humaniste aura consacré la quasi-totalité

52. Lohans mourut en 1493. Reuchlin lui vouait une estime profonde. Il le qualifie de « præceptor meus, mea sententia valde doctus homo Jacobus Jehiel Loans Hebræus» (Rudimenta Hebraica, p. 249) et de «humanissimus præceptor meus homo excellens » (Ibid., p. 619). On connait fort peu de choses de sa vie et Ludwig Geiger pensait (Johann Reuchlin, sein Leben und seine Werke, Leipzig, 1871, p. 106) qu'il servit de modèle au juif Simon du De arte cabalistica publié par Reuchlin en 1517.

53. Abdia Sforno (1470-1550) reste connu comme l'un des plus importants commentateurs de la Torah de son époque. 
de ses publications - quand ce n'est pas à la défense de ces mêmes Lettres hébraïques contre les dominicains, pendant le Bücherstreit.

On peut se demander pourquoi il tient à préciser que ses deux parents sont chrétiens ( Georges et Elissa, citoyens de la ville de Pforzheim »). C'est sans doute pour montrer que c'est la première fois que l'hébreu sera enseigné par un maître qui n'est pas juif de naissance, comme le furent Nicolas de Lyre et Paul de Burgos.

\section{LE DÉGOÛT DES VERNACULAIRES}

Les deux premiers livres ayant exposé les rudiments des lettres et des mots, le troisième va aborder la grammaire, c'est-à-dire de la construction du discours, donc la langue proprement dite. À ce point, Reuchlin tient à répondre par avance à l'accusation de ceux qui lui reprocheront d'être un barbare au motif qu'il ne fait pas partie de ceux qui sont supposés parler latin dans l'exercice de leur profession, c'est-à-dire des clercs. Il n'est pas professeur d'université, ni théologien, ni même juriste de droit canonique. Il n'est que juriste civil et rend donc la justice en allemand : "Qu' on ne me reproche pas de parler vernaculaire aux indigènes. ${ }^{54}$ »

Sa justification consiste en un vaste tableau littéraire aux couleurs fort humanistes dans lequel il s'introduit sous les traits de Ménélas interpellé par Tyndare (faisant observer au passage qu'il sait distinguer le personnage de l'auteur et que c'est donc Euripide qui parle par la bouche de Tyndare, cité en grec dans le texte): «Tu es devenu barbare depuis le temps que tu as séjourné chez les barbares. ${ }^{55}$ »

Il riposte en se représentant sous les traits de Diogène, qui se protège de la barbarie allemande dans son tonneau grec.

« Je me réfugie donc tous les jours, tout seul dans ce tonneau de Diogène, car je ne trouve dans mon entourage personne qui accorde un prix à l'élégance des mots, ni à l'étude de l'éloquence, mais seulement des paysans et des prêtraillons. Quant à ceux qui me rendent visite, comme

54. [...] non mihi vitio dabunt, quod vernis vernacule loquor (Rudimenta, p. 546).

55. Euripide, Oreste, v. 485. 
il arrive tous les jours, ils ne me consultent pas sur la production de la pourpre ni de l'or dont on fait les couronnes, mais sur des affaires d'égouts, de marais, de bois, de chemin ou d'autres querelles vulgaires, tant au tribunal qu' au civil. Et je dois leur répondre sur le droit en vertu de mon métier, en ne me conformant pas à l'usage de la langue latine, mais souabe, et il n'y a pas de misère plus grande, mon frère, que de quitter les prairies verdoyantes pour les rues crasseuses. ${ }^{56}$ »

Cette image lui permet d'évoquer ses souvenirs littéraires et de constater que les meilleurs auteurs (antiques par définition) n'ont pas dédaigné de se moquer des vernaculaires. Excellente occasion de citer les profanes Aristophane, Caton et Plaute. Ce premier niveau de moquerie ne porte même pas sur le contenu du discours, mais sur les sons eux-mêmes : quels barbares que ces gens dont la langue est incompréhensible !

Mais il est difficile d'en rester à un niveau d'argumentation aussi rudimentaire et Reuchlin monte à un ton plus élevé pour confondre ses détracteurs en faisant appel à trois couples antinomiques classiques :

«Je pense pourtant qu'il va arriver - de peur que son Thersite ne manque à Agamemnon, Zoïle à Homère, les Nuées à Socrate - que les médisants fanatiques s'attaquent aussi à moi un jour. ${ }^{57}$ »

Thersite est le héros ridicule de l'Iliade, le plus laid de tous les Grecs, boiteux, bossu, chauve et lâche. Mais il est surtout l'insulteur d'Agamemnon (II, 211). Zoïle d'Amphipolis est un personnage historique. C'est le fameux critique du $I^{\mathrm{e}}$ siècle qui reprocha à Homère ses affabulations. Il était surnommé Homeromastix (« le

56. Solus ergo quom in hoc Diogenis dolio totos dies delitescam, nec mecum vivat ullus hominum, cui verborum elegantia et eloquentice studium sit in pretio, sed agricola tantum et sacrificuli, aut si qui accedant, ut fit quotidie, qui me non de murilegulis, nec auro coronario, sed de cloacis, paludibus, ligno, itinere, alliisve popularibus jurgiis, tam pratoriis, quam civilibus consulunt, quibus de jure pro mea professione respondere debeam, non Latinorum, sed Suevorum observato dicendi usu nihil ultra miseris, mi frater, me post virentia prata iam scabros calles ambulare (Rudimenta, p. 546).

57. Hoc unum tamen esse futurum arbitror, ne desit Agamemnoni suus Tersites Homero Zoilus, Nephela Socrati, quod etiam contra me quoque prodeant aliquando phanatici quidam susurrones (Rudimenta, p. 547). 
fouetteur d'Homère »). Quant aux Nuées pour Socrate, cette allusion à la comédie d'Aristophane peut se comprendre dans un double sens, selon qu'il s'agit du chœur des Nuées proprement dites, ou bien de la comédie elle-même. Les Nuées (personnages) se présentant plutôt comme des protectrices de Socrate, c'est sans doute de la comédie qu'il s'agit, en tant que critique excessive de Socrate.

Si l'on suit ces trois comparaisons, Reuchlin se présente successivement dans les rôles d'Agamemnon, d'Homère et de Socrate, tous les trois victimes de critiques injustes. On observera qu'Homère est sans conteste le prince des rhéteurs (ou à tout le moins celui des poètes) et Socrate celui des philosophes. Mais Agamemnon n'est sûrement pas celui des grammairiens.

\section{L'AUTODIDACTE CRITIQUE}

En tout état de cause, que lui reprocheront ses détracteurs? Ils le jugeront d'abord incapable d'avoir réussi à apprendre l'hébreu tout seul et à un âge avancé.

« Ils diront en effet que je n'ai pas pu parvenir sans précepteur à un tel sommet de la lecture de l'hébreu dans mon âge avancé, quels que soient mes efforts, au point que mes études soient utiles aux autres. ${ }^{58}$ »

La question de l'apprentissage des langues, et de l'hébreu en particulier, redevient donc centrale. Reuchlin leur oppose d'abord quelques illustres devanciers qui ont réalisé cet exploit :

« Je leur opposerai vigoureusement Origène, saint Jérôme et le fameux Nicolas de Lyre ${ }^{59}$, dont il est prouvé qu'ils se sont tous mis à l'hébreu à l'approche de la vieillesse. Et qui n'a pas illustré l'Église orthodoxe, après les apôtres, dans les Lettres hébraïques plus solidement que tous

58. Dicent enim non me potuisse in senilibus annis absque praceptore etiam qualicumque conatu ad tantum hebraica lectionis fastigium ascendere, ut studia mea ceteris prosint (Ibid.).

59. Ce fleuron des théologiens franciscains (1270-1340) était réputé fils de conversos. 
les autres, sinon Paul de Burgos ${ }^{60}$, ce maître remarquable qui a entrepris de les étudier à un âge plus tardif $?^{61}$ »

Reuchlin retourne ensuite cette accusation à son avantage en rappelant qu'il n'a pas étudié les langues dans leurs pays d'origine ni dans des écoles publiques - donc bon marché. Il n'a pas étudié le grec en Grèce chez Jonas (dont nous ignorons l'identité), mais à Bâle, Paris et Rome. Quant à l'hébreu, il ne l'a pas étudié à Jérusalem «ni dans les synagogues publiques des juifs » (neque in publicis Judaorum synagogis - terme désignant sans doute les écoles talmudiques, Schul en pays germaniques), mais à Linz et à Rome. De plus, il a étudié ces deux langues auprès de maîtres privés, donc coûteux, si bien qu'il a dû se contenter de miettes, comme Lazare se nourrissant à la table des riches. Il a payé quatre maîtres pour le grec (Andronicos Contoblacas à Bâle, Georges Hermonyme à Paris, Jean Argyropoulos à Rome, Demetrios Chalcondylas à Florence et à Milan) et deux pour l'hébreu (Jakob Jehiel Lohans à Linz et Abdias Sforno à Rome). Cet argument n'est pas superflu pour la promotion du présent traité, en démontrant qu'un homme motivé peut parfaitement apprendre l'hébreu à l'aide d'un bon manuel.

Mais le reproche principal contre lequel Reuchlin doit se défendre maintenant est celui de corriger la Vulgate :

«Mais je crois que les jaloux s'insurgeront davantage contre notre Dictionnaire, dans lequel nous critiquons souvent les traductions. “Quel crime ! s'exclameront-ils. Il n'y a rien de plus indigne à l'égard de la mémoire de nos pères! Aucun crime plus cruel que le fait que cet homme essaye de ruiner des personnages si saints et remplis de l'esprit divin! L'écriture du très saint Jérôme, comme en témoigne le pape Gélase, est reçue dans l'Église. Le vénérable père Nicolas de Lyre, qui

60. L'érudit juif Salomon Ha Levi s'était converti vers l'âge de quarante ans avec toute sa famille (sauf sa femme). Il devint dominicain sous le nom de Paul de Santa Maria et mourut en 1435, après avoir occupé les plus hautes responsabilités politiques dans le royaume de Castille. Il fut notamment chancelier et archevêque de Burgos.

61. Quibus statim Origenem objiciam, sanctumque Hieronymum et illum de Lyra Nicolaum: qui omnes appropinquante senio primum hebraicis studuisse probantur. Quin potius nemo ferme omnium post apostolos orthodoxam ecclesiam hebraicis literis illustravit, qui non eas in atate provecta discere cœeprit, prater insignem magistrum illum Paulum episcopum Burgensem (Rudimenta, p. 547). 
a exposé la Bible à tous les fidèles du Christ, est prouvé un homme très honnête. Et voilà que se lève je ne sais quelle Petite fumée ${ }^{62}$, qui affirme que dans beaucoup de passages il a traduit faussement." 63 »

Pour s'en justifier, il utilise un argument indirect qui lui évite d'attaquer Jérôme frontalement. Il remonte la chaîne des commentateurs de la Bible, dont chacun a critiqué le précédent, au nom de la veritas hebraica. Le plus récent est Matthias Döring, qui a critiqué Paul de Burgos, qui a critiqué Nicolas de Lyre, qui a critiqué Jérôme, qui avait lui-même critiqué les Septante et saint Paul. Ainsi, la critique de la Septante par Jérôme légitime celle de la Vulgate par Reuchlin. Si bien que ce dernier, ayant bénéficié des leçons de ses maîtres juifs Lohans et Sforno (« les plus sages des Hébreux »), peut maintenant s'autoriser à critiquer tous ses prédécesseurs. Sa plaisanterie finale sur Jérôme, ironiquement paraphrasée d'Aristote (amicus plato sed magis amica veritas), est explicite :

«Bien que je vénère saint Jérôme autant qu'un ange et que je respecte [Nicolas de] Lyre comme un maître, j'adore cependant la vérité comme un dieu. ${ }^{64}{ }^{»}$

\section{MODÈLES ET CONTRE-MODÈLES}

En achevant la lecture de ces trois textes qui enserrent les Rudimenta hebraica, on observera que la liste des personnages cités à des titres divers par Reuchlin comme modèles - à l'exception de ses professeurs - est assez hétéroclite : Ulysse, pour sa résistance aux Sirènes; Opilius, Atéius philologus et Valérius Caton, pour

62. Le nom de Reuchlin signifie littéralement « petite fumée » (Räuchlein) en allemand.

63. At gravius insurgent, credo, invidi contra Dictionarium nostrum in quo multorum frequenter interpretationes taxantur " Proh scelus exclamabunt, nihil indignius patrum memoria, nihil admissum crudelius, cum ille homo audacissimus tot et tam sanctos viros divino spiritu afflatos labefactare contendat. Hieronymi beatissimi scriptura, Geladio Papa teste, recepta est in ecclesia, venerabilis pater Nicolaus de Lyra ordinarius expositor Biblia omnibus Christi fidelibus vir integerrimus probatur. Jamjam exortus est aliquis fumulus, qui plurimus in locis illos imperite transtulisse notat » (Rudimenta, p. 548).

64. Quamquam enim Hieronymum sanctum veneror ut angelum et Lyram colo ut magistrum, tamen adoro veritatem ut deum (Rudimenta, p. 549). 
leur cursus ; Euripide, Diogène, Aristophane, Caton et Plaute, pour l'élégance de leur langue ; Ménélas ( $v s$ Tyndare), Agamemnon ( $v s$ Thersite), Homère (vs Zoïle) et Socrate, pour leurs détracteurs ; Origène, saint Jérôme, Nicolas de Lyre et Paul de Burgos, pour l'âge avancé auquel ils ont étudié l'hébreu ; Pic de la Mirandole et Nahmanide pour leur profondeur.

La lecture de cette liste amène quelques observations. Premièrement, les païens de l'Antiquité (pour un homme de la Renaissance, Ulysse et Agamemnon étaient des personnages historiques) sont largement présentés comme modèles positifs. Sur les dix-sept nommés, seuls deux sont critiquables : Thersite et Zoille. La proportion est inverse chez les chrétiens.

En face d'eux, les Pères et docteurs de l'Église brillent par leur absence - à l'exception d'Origène, de Jérôme, de Nicolas de Lyre et de Paul de Burgos. Parmi eux, deux conversos et un seul qui échappe à la critique (Origène). Les chrétiens ne sont cités que pour leurs mauvaises connaissances linguistiques, la palme revenant à Jérôme, convoqué comme témoin à charge contre l'apôtre Paul, lequel est cité pour l'inélégance de son grec... et Reuchlin réussit l'exploit de ne pas le nommer par son nom («l'apôtre ») :

«Il [Jérôme] a écrit que l'apôtre a ignoré les règles de la grammaire dans l'avant-avant-dernière question de la lettre à Hédibia. ${ }^{65}$ »

Voici ce que Jérôme avait écrit dans la lettre en question :

«Quoique Paul eût la science des saintes écritures et qu'il ait possédé la grâce de parler diverses langues [...] il ne pouvait cependant pas exposer d'une manière convenable la majesté du texte sacré avec l'élégance de la langue grecque. Il avait donc Tite pour interprète [...]. ${ }^{66}$ »

À travers Jérôme, qui a reconnu lui-même ses lacunes en hébreu (il est nommé à neuf reprises, alors que Nicolas de Lyre ne l'est que cinq fois), c'est donc clairement l'auteur de la Vulgate qui est visé.

65. Quod apostolum notaverit imperitum artis grammatica in epistola ad Hedibiam, quastione antepenultima (Rudimenta, p. 549).

66. Cumque haberet scientiam sanctarum scripturarum, et sermonis diversarumque linguarum gratiam possideret [...] divinorum sensuum majestatem digno non poterat graci eloquii explicare sermone. Habebat ergo Titum interpretem [...] (Jérôme, Lettre CXX à Hédibia, chap. X). 
Au final, seuls trois personnages échappent totalement à la critique et leur réunion a de quoi surprendre. Le premier est Origène, dont la réputation d'hétérodoxie n'est pas un mystère. On sait à quel point ce personnage a fasciné tous les humanistes et en particulier Érasme, pour avoir composé les Hexaples, c'est-à-dire pour avoir cherché à rendre compte de l'ensemble des traditions philologiques de la Bible, tant en grec qu'en hébreu - mais pas en latin. Son platonisme, qui l'avait précisément rendu suspect aux « orthodoxes », le rendait sans doute d'autant plus sympathique aux humanistes, surtout depuis l'épisode de l'Académie de Careggi.

Le second est Pic de la Mirandole, auprès duquel Reuchlin s'était initié à la Kabbale en 1490 :

« [...] Comme l'atteste Jean Pic de la Mirandole, ce noble et généreux comte de l'Empire romain et philosophe, quand il dit dans ses conclusions : "Celui qui connaîtra l'organisation de la langue hébraïque profondément et radicalement, et qui saura la reporter selon les mêmes proportions dans les connaissances, celui-ci possédera parfaitement la norme et la règle pour découvrir tout ce qui est connaissable". ${ }^{67}$ »

Ses démêlés avec Rome et sa condamnation en 1487 étaient également de notoriété publique. Reuchlin, qui l'avait connu, était particulièrement sensible à cette affaire car, quatre ans plus tard, dans son Rapport à l'empereur sur la confiscation des livres des juifs ${ }^{68}$, il rappela que Sixte IV avait approuvé les conclusions de l'Apologie de Pic sur la Kabbale.

Le troisième est Nahmanide de Gérone ${ }^{69}$, le kabbaliste juif, héros en 1263 de la fameuse dispute de Barcelone contre le dominicain converso Paul Christiani.

«C'est pourquoi Rabbi Moïse, fils de Nahman de Gérone, dans son introduction à ses commentaires de la Genèse entreprend de prouver

67. [...] Testis ille imperii Romani nobilis et generosus comes Joannes Picus Mirandulanus, Philosophus in suis conclusionibus, quum ait, "Qui ordinem hebraica linguce profunde et radicaliter tenuerit atque illum proportionabiliter in scientiis servare noverit, cujuscunque scibilis perfecte inveniendi normam et regulam habebit» (Rudimenta, p. 620).

68. Ratschlag ob man den Juden alle ire bücher nemmen, abthun und verbrennen soll (1510), repris dans son Augenspiegel de 1511.

69. Connu sous le nom de Ramban. 


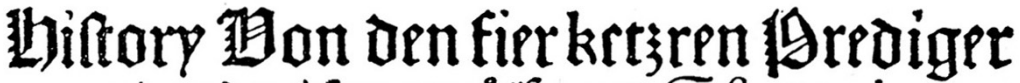
ozdens der obferuang jư 25 ern im Griweyser land verbzant/ in dé jar nork $\mathbb{C}$ raiftigeburt. (D. $\mathbb{C}$

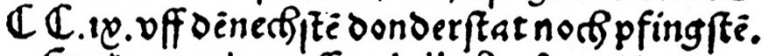
Ein Eurser begriff onbellicher frcuel hano lüg Bodiftrats / ODurnars / Docroz

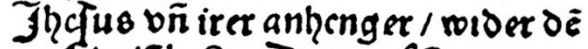
Cl)ziftirfen Doctoz BDatun Zuther/bon alle liebl)aber Euangchifther lere.

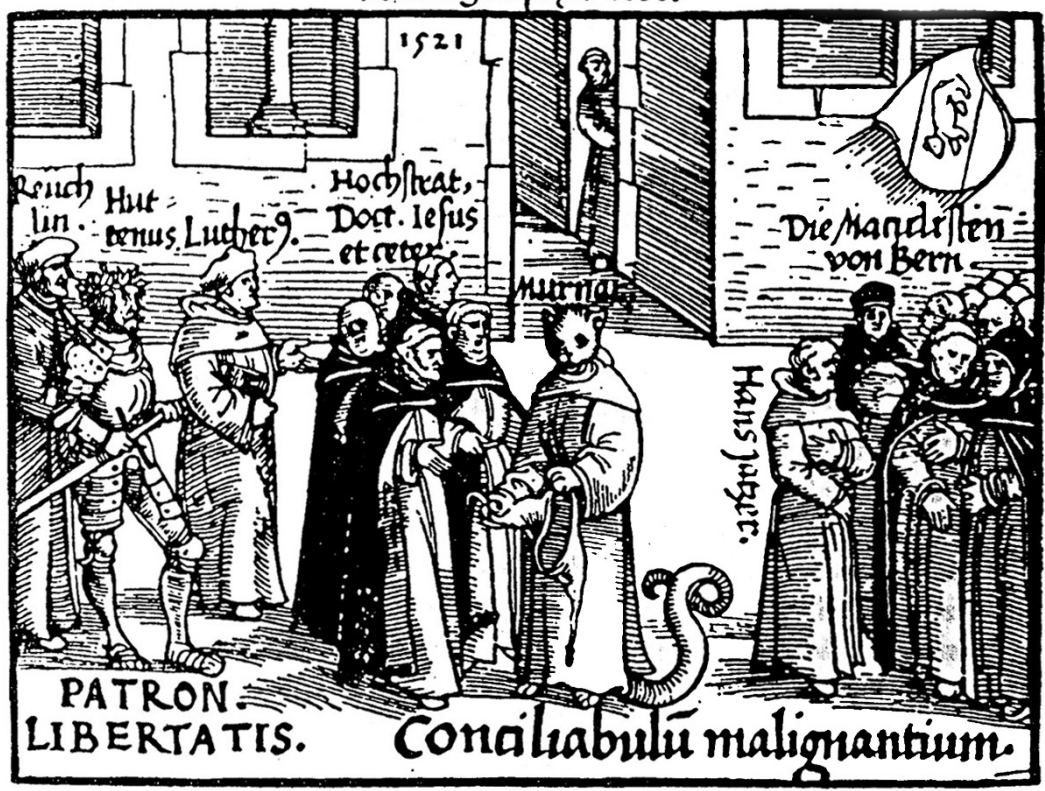

Jean Reuchlin (à l'extrême gauche de la gravure), qualifié en 1521 de «patron de la liberté » (Patron libertatis), aux côtés du chevalier Ulrich von Hutten et de Martin Luther, les deux grands pamphlétaires antipapistes.

Cette illustration de 1521 orne la page de titre d'un libelle publié par le franciscain Thomas Murner (représenté en chat au milieu de la gravure) contre l'imposture des faux miracles de Berne de 1509. Quatre dominicains (à droite de l'image) avaient été impliqués dans l'affaire et avaient fini sur le bûcher. C'est le seul portrait contemporain de Reuchlin connu à ce jour. (Max Brod, Johannes Reuchlin und sein Kampf, Stuttgart, Kohlhammer, 1965, ill. ${ }^{\circ}{ }^{8}$.) 
par un long discours, que le roi Salomon a puisé tout le flux de sa sagesse dans les lettres du Pentateuque. ${ }^{70}$ »

En se réclamant de tels modèles, Reuchlin se posait à nouveau avec ostentation en marge de ses coreligionnaires. Cela explique sans doute combien il les irritait et devint si aisément leur bouc émissaire, lors de l'affaire des livres juifs.

Au terme de cette lecture, on peut se demander si la complexité des détours stylistiques empruntés par Reuchlin ne révèle pas combien ce personnage se pliait malaisément aux catégories intellectuelles et sociales de son époque. Ainsi, il se présentait comme juriste chez les théologiens, théologien chez les poètes, professeur hors de l'université, hébraïsant chez les latins, Grec chez les Allemands, kabbaliste chez les chrétiens, chrétien chez les juifs et néanmoins poète à ses lecteurs.

On notera enfin que, dans les dernières lignes de sa lettre, il reproche à ses « compatriotes » (nostrates) juifs de refuser d'enseigner leur langue aux chrétiens. Et pourtant, quelques années plus tard, lors des persécutions de Cologne, c'est en tant que « concitoyens » (concives) de l'Empire qu'il allait les défendre - mais c'est là une autre affaire.

18, Cité des fleurs

75017 Paris

saladin-jc@noos.fr

70. Unde Rabi Moyses, filius Neheman Gerundensis in exordio Commentariorum Geneseos, longo sermone probare conatur, Salomonem regem omnes sapientice suce influxus, de Pentateuchi literis hausisse (Rudimenta, p. 620). 